The patient returned on July 21st, 1924, as the trouble was, she said, recurring. Examination revealed a very slight swelling of the left lacrymal gland but there was no narrowing of the orbital fissure, and no diplopia. There was an appreciable fullness of the upper half of the right parotid gland over an area $3 \mathrm{~cm}$. in diameter. The left submaxillary gland was no longer palpable. The right lacrymal, right submaxillary, and left parotid glands remained unaffected. The treatment was repeated from July 21st to $25 \mathrm{th}$, and she was asked to report early in October. She did not come on the appointed date, but wrote saying that she was an in-patient at King's College Hospital, suffering from a tumour of the spleen, but that her face was quite well. She was under the care of Sir Charlton Briscoe, whose house-physician, in reply to an inquiry as to the patient's condition, wrote :

"The patient was admitted here on August 25th, 1924 . Spleen greatly enlarged, lower border below level of umbilicus, consistence hard, surface slightly uneven, edge sharp, not tender. Live enlarged (lower border 2 inches below right costal margin), hard sharp edge, not tender. Ascites definitely present, but not marked. No oedema of feet; urine loaded with urates, no albumin. Wassermann test positive. The salivary and lacrymal glands appear to be quite normal at present.

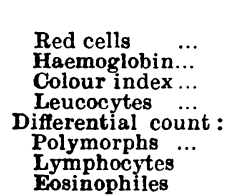

$$
\begin{array}{ccccc}
\multicolumn{3}{c}{\text { Blood }} & \text { Count. } \\
\ldots & \ldots & \ldots & \multicolumn{2}{c}{3,440,000 \text { per c.mm. }} \\
\ldots & \ldots & \ldots & \ldots . & 68 \text { per cent. } \\
\ldots & \ldots & \ldots & \ldots & 1,600 \text { per c.mm. } \\
\ldots & \ldots & \ldots & \ldots & 1,600 \\
\ldots & \ldots & \ldots & \ldots & 42 \text { per cent. } . \\
\ldots & \ldots & \ldots & \ldots & 56 \%, \\
\ldots & \ldots & \ldots & \ldots & 2
\end{array}
$$
The case is regarded as one of splenomegaly of the Banti type,
associated with and probably irritated by a syphilitic infection."

Case 2.

A married woman, aged 41, was sent by Professor J. B. Bradbury of Cambridge to the Radium Institute for treatment. The disease had existed for about seven years, and had been slowly progressive. Some $x$-ray treatment had been given during 1925 , with slight but transient benefit, and as the last exposures had failed to produce any improvement, radium treatment had been suggested.

When examined on March 10th, 1926, both parotids were found to be enlarged, each to the size of a small lemon, the covering skin was smooth and not implicated, and the swellings were firm skin was smooth and not implicated, and the swellings were firm
and elastic. Some degree of movement of both parotids was and elastic. Some degree of movement of both parotids was
possible. Both submaxilary glands were enlarged to the size of possible. Both submaxillary glands were enlarged to the size of the mouth. They presented the same physical characteristics as the parotid glands. The palpebral glands were not definitely enlarged, but the mucosa of both upper eyelids. was much congested, and the eyes watered a great deal. The salivary secretion was very deficient, and the patient complained of great and persistent dryness of the mouth. There was no history of syphilis. Treatment was carried out by means of numerous half-strength applicators containing in all $240 \mathrm{mg}$. of radium element. These were screened with $2 \mathrm{~mm}$. of lead, and so disposed as to procure an effective " crossfire" radiation. The total exposure was of thirty hours' duration, given at spaced intervals over a period of three days.

The patient came again for examination on May 27th, 1926. No trace of the swellings remained, and the flow of saliva had returned. She stated that the swellings began to diminish rapidly a few days after treatment, and had quite disappeared within three weeks. Wholly disinterested and unsolicited testimony to the efficacy of the treatment was afforded by the patient's son, a child of 8 , who, gazing at his mother in some bewilderment a few of 8, who, gazing at his mother in some bewilderment a few
face has got so thin!"

The disease is rare, but cases are reported from time to time by competent observers. No specific line of treatment has ever been indicated; arsenic and iodides have been the drugs chiefly employed, and in some instances $x$ rays have been found to be of service.

The cases here recorded are not absolutely typical, but it would appear difficult to classify them as anything other than Mikulicz's disease.

In both instances the condition was bilateral, but in one the lacrymal glands were not definitely attacked. In the other the disease was asymmetrical, affecting the lacrymal and submaxillary gland on one side, and the parotid gland only on the other. One patient had a definite syphilitic history, and the Wassermann reaction was strongly positive. The other patient had enjoyed good general health all her life.

The extremely rapid response to the treatment is undoubtedly due to the pathological nature of the malady -namely, an intense lymphocytic infiltration of the sustentacular tissue of the glands; the lymphocyte being of all cells the most susceptible to radium irradiation.

\section{A NEW METHOD OF PREPARING TUBERCULIN.}

BY

H. HYSLOP THOMSON, M.D., D.P.H.,

COUATY TUBERCCLOSIS OFFICER AND COUNTY AIEDICAL OFYICER OF HEALTH FOR HERTS.

THE conclusions and recommendations in the following paper are based on the experience of tuberculin treatment in two series of cases, including both sanatorium and dispensary patients, during a period of ten years. In my experienco tuberculin has proved of definite value in the treatment of certain forms of tuberculosis. When used in the sanatorium at the stage when the patient has become nonreactive to manual labour it provides a useful guide to the absence or presence of susceptibility on the part of the patient to relapse from overstrain, and, if tolerance has been established to increasing doses, it secures a definite although admittedly not a permanent protection against such relapse. In non-pulmonary tuberculosis the beneficial effect of tuberculin is frequently quite definite. Old tuberculous sinuses, discharging tuberculous glands, and localized lesions of the skin which have resisted other forms of treatment frequently yield in quite a striking manner to treatment with tuberculin.

In acute and active cases of the disease tuberculin has proved to be of little value, and is indeed contraindicated because of the disturbance to which it gives rise. In the less active or smouldering type of the disease, however, in which the temperature does not exceed $100^{\circ} \mathrm{F}$., treatment with tuberculin TOA (filtrate) does in certain cases givo good results by lowering the temperature and lessening the swing. In such cases treatment with tuberculin should be combined with strict rest in bed, but even in patients of this type who have not been confined to bed the beneficial effect of tuberculin TOA on the temperature has been noted.

The general conclusions regarding the value of tuberculin treatment are as follows: (a) Tuberculin is of no value, and is indeed contraindicated in the treatment of acute and active cases of pulmonary tuberculosis. (b) Inoculation with tuberculin TOA (filtrate) can safely be given in certain cases of subacute type in which the temperature does not exceed $100^{\circ}$ F., and is indeed not infrequently followed by beneficial results, as is indicated by a lowering of the temperature to within normal limits. (c) Inoculation with tuberculin BE or TR containing the body of the bacillus or its contents is of value in ambulant cases of pulmonary tuberculosis which do not react to the muscular movements associated with walking exercise and manual work. (d) Tuberculin treatment induces complete healing in certain cases of non-pulmonary tuberculosis, especially those associated with a chronic discharging sinus. (e) Tuberculin treatment rarely promotes complete healing in. pulmonary tuberculosis, but if used for an extended period in suitable cases it tends to induce quiescence, to encourage fibrosis, and to confer some definite protection against relapse.

It is doubtful whether the treatment of tuberculosis with tuberculin has received a sufficiently fair and extended trial in this country. The variety of tuberculins in use and the different methods of administration employed have militated against accurate treatment and satisfactory results. Too frequently treatment has been employed in quite unsuitable cases or has been discontinued because no immediate striking result has been obtained. In the preparation of tuberculin also methods have becn adopted which are no doubt partly responsible for tho absence of more definite and more immediately beneficial results. These methods are $(a)$ the selection of an unsuitable strain of the tubercle bacillus as a basis for preparation; (b) the continued culturing of strains of tubercle bacilli on artificial media which differ in many respects from the natural medium found in the human or bovine host, with the result that an artificial strain of bacillus has been evolved; and (c) the employment of heat and chemical or physical agents for purposes of sterilization and extraction, which agents must modify the character of the toxic products of the bacilli elaborated in the culture medium during the process of growth. 
The general principles underlying the following method of preparation are the employment of a culture medium containing blood serum unsterilized by heat, so as to provide a medium as nearly as possible similar to that which obtains in the human body, and the inclusion of part of the filtrate in successive subcultures. It is necnssary to emphasize the importance of several factors which are regarded as essential to the preparation of the tuberculin. These are (a) the preparation of a suitable culture medium containing sterile but unheated blood serum of human origin for bacilli of human type and of bovine origin for bacilli of bovine type; (b) the employment of a strain of human and bovine bacilli obtained from an acute or systemic type of the disease; (c) the evolving from this by culturing and subculturing of a strain of tubercle bacilli which will grow actively on the media of human and bovine material before proceeding with the preparation of the tuberculin; and $(d)$ the evoidance in the preparation of the tuberculin of any measures which are likely to interfere seriously with the character of the final filtrate.

The general technique of the method recommended in the preparation of tuberculin of human origin is as follows:

(a) The growth of the special strain of tubercle bacilli on a medium containing unheated blood serum. The morphological, cultural, staining, and pathogenic characters of the bacilli and the toxic and infective properties of the filtrate to be investigated.

(b) A subculture from (a) on a medium comprised of unheated blood serum and an equal portion of the filtrate from $(a)$. A similar line of investigation to be carried out.

(c) A subculture from $(b)$ on a medium comprised of unheated blood serum and an equal portion of the filtrate from $(b)$.

(d) A subculture from (c) on a medium comprised of unheated blood serum and an equal portion of the filtrate from $(c)$.

The extent of subculturing will depend upon the growth obtained and the results of the investigations as to the character of the bacilli and the filtrates in the various subcultures. In the method described above the inoculum is taken from each previous culture, but an alternate series of experiments would be necessary in which a fresh inoculum from the original strain on each of the successive series of media is employed. The possibility of stimulating the growth of the bacillus in the various subcultures by oxygen or other means should be considered. The tuberculin, when completed, will consist of two portions: (a) the final filtrate, and (b) the final filtrate combined with an emulsion of bacilli from the final culture. The final filtrate should not be sterilized by heat but.by repeated passing through a Chamberland filter, and experimental work would be necessary to prove that it does not possess pathogenic properties. Should experiments prove that in spite of repeated filtration it possesses pathogenic or infective characters, sterilization by some other means, such as by exposure to ultra-violet rays, must be considered. The tubercle bacilli forming the emulsion would require sterilization by heat before the final filtrate is added to constitute $(b)$. The keeping properties of both $(a)$ and $(b)$ should be tested by prolonged incubation.

The aim of the above investigation is twofold. In the first place to obtain and to test a tuberculin composed of reinforced filtrate alone and of the same filtrate in combination with the bacillary contents prepared under conditions as nearly as possible approximating those under which the bacillus grows and develops in the human body. Secondly, to throw some further light on the life-history of the tubercle bacillus and on the character of the toxins elaborated by the organism in the process of growth under natural conditions.

If we attempt to visualize the life-history of the tubercle bacillus in the human body we are forced to accept the following conclusions: (a) That in the process of development and multiplication the bacillus at the outset elaborates exotoxin, and that it grows and matures on a medium of gradually devitalized human tissue and blood lymph which contains exotoxin. (b) That at some period during the life-cycle of the bacillus endotoxins are set free either by a process of extrusion in consequence of interference with the integrity of the fatty envelope or by a destructive process arising from the action of bacteriolysins or autolytic enzymes. The clinical features in the early and more advanced stages of tho disease can only be explained by some such conception with wide variations in the conditions required for the development and completion of the process.

In January, 1924, I submitted an outline of the method described above for the preparation of a tuberculin to Professor Tulloch of St. Andrews University, who kindly arranged for Dr. Cumming, who was working in his bacteriological department, to make a preliminary investigation as to the possibility of preparing a tuberculin by the method suggested. Professor Tulloch thought that before proceeding to investigate the growth on human media--the constituents of which are difficult to obtain-a technique should be elaborated on bovine material. The main results of the preliminary investigation by Dr. Cumming are briefly as follows.

"1. It has been found that even strains of tubercle bacilli which have become accustomed to glycerinated media, both bovine and human, grow reasonably on 1-4 non-heated sterile rabbit plasma with no addition of fresh tissue and no glycerin.

2. A very careful aseptic technique was elaborated for the growth of bovine tubercle bacilli on a cone-shaped island of fresh ox heart one inch high and three-quarters of an inch in diameter at the base, in a layer of prepared ox heart extract from one to one and $a$ half centimetres in depth in specially made bottles.

3. In the above and subsequent experiments different dilutions of serums and different diluents (saline, Locke's solution, etc.) were used along with an inoculum of human and bovine strains from human lesions and bovine strains from bovine lesions with practically always perfect results as far as asepsis was concerned,
but always with disappointing results as regards growth of the tubercle bacilli.

4. The reasons for these disappointing results appeared to be due to the fact that when these bottles of serum and tissue were incubated autolysis of the tissue took place, with the result that the growth of the tubercle bacilli was thereby inhibited.

5. In ono series of experiments growth (of a kind) was obtained in four successive generations, using in each case equal parts of fresh medium and filtrate from the previous batch. In thes it was shown that the tubercle bacilli in the fourth generation had not been altered as regards morphology, staining reaction, cultural reactions, or pathogenicity, and that the final filtrate had only very slight antigenic properties, as evidenced by Koch phenomenon.
. It was further found that the strain of tubercle bacilli 6. It was further found that the strain of tubercle bacilli
employed did not grow so well on fresh human serum sterilized by candlings as was expected. Dr. Cumming was not able to satisfy himself that this was due entirely to the fact that the strains had been cultivated practically exclusively on glycerinated medium."

In the above summary of the results obtained in the preliminary investigation the one difficulty which at the outset has militated against success has been inability to obtain a vigorous growth of tubercle bacilli on sterile but unheated media of human and bovine material. In one series of experiments with a bovine strain and bovine material a somewhat feeble growth was obtained in four successive generations, using in each case equal parts of fresh medium and filtrate from the previous culture. The fact that such growth was obtained indicates the desirability of further investigation as to the possibility of obtaining a more vigorous growth on successive filtrate media.

The difficulty experienced in obtaining an active growth of tubercle bacilli on fresh human serum is significant and tends to support the thesis submitted in this paper. It emphasizes the necessity of further investigation in the direction of elaborating suitable unheated culture media of both human and bovine material with a view to the evolution of a special strain of tubercle bacilli which will grow vigorously on such media. The question of material of human origin for the preparation of suitable media may present some difficulty, but in the healthy human placenta there is to be found an inexhaustible source of supply.

A further important difficulty met with in the experiments was the inhibition of the growth of the tubercle bacilli in the bovine medium by the occurrence of autolysis of the tissue constituting part of the medium. This was no doubt due to the action of autolytic enzymes in the sterile but unsterilized cone of ox heart in the presenco of unheated heart extract or blood serum. Sterilization of this tissue before use as an island for inoculation would prevent autolytic action taling place and would not seriously interfere with the experiment.

Owing to the absence of facilities it has not been found possible to continue this investigation beyond the point 
described above. I submit the following propasals for further investigation:

1. The preparation of suitable media of human and bovine material containing unheated bloos serum.

2. The evolving of a vigorous strain of tubercle bacilli which will grow on human and bovine media which are not sterilized by heat.

3. The elimination of autolysis in such media and investigation into the possible action of autolytic enzymes on the tubercle

4. The preparation of a tuberculin by the method described

5. Preliminary investigations to be carried out with bovine tubercle bacilli on media of bovine material.

In conclusion I have to express my thanks to Professor Tulloch for his valuable advice and assistance, and to $\mathrm{Dr}$. Cumming for the time and labour he has given to the preliminary investigation.

\section{A CASE OF TRANSVERSE ECTOPIA OF THE TESTIS.}

\section{BY}

\section{A. GRAY BANKS, M.D.Glasg., F.R.C.S.ED.;} SURGEON, EAST SUFFOLK AND IPSWICH HOSPITAL.

THE interest of the following case is increased by the note Sir Arthur Keith has been good enough to append.

A man, aged 28, married three years, no children, was admitted to the East Suffolk and Ipswich Hospital in December, 1925, with a painful swelling in the left groin. Eighteen years earlier an operation for left inguinal hernia had been performed in the same hospital, but details are unobtainable. He had been aware of the lump ever since the operation and had noticed it becoming gradually larger, more superficial, and moving downwards. Nine graduaty larger, more superficial, and moving downwards. Nin months ago had an attack of acute pain afting a heavy weight, and the lump became tender and swollen, and had remained more or less tender since. Two further attacks of pain occurred, one three months, and the other three weeks ago. O each occasion micturition was frequent for about two days; between the attacks there were no urinary symptoms. Sexual
power developed when he was about 14, and has been normal since.

He was a spare, vigorous, active-looking man of good intelligence; the secondary sexual characters were well developed A linear scar of old standing was present over the left inguinal canal, and lying at the external ring just below the pubic spine was a firm ovoid swelling about the size of a walnut, slightly movable and tender on pressure. The scrotum was small and contained only one testis (left) from.which an easily palpable cord could be traced upwards. The right side of the scrotum was empty and no opening could be felt at the usual site of the external ring opening could be felt at the usual site of the confirmed by intra-abdominal palpation during the operation).

\section{Operation.}

An oblique incision was made over the inguinal canal and the swelling identified as a testis (although at first sight the appearance suggested an ovary). It had a well marked tunica vaginalis. From the upper border of the testis a fold of fascia and peritoneum, forming a definite mesorchium and having a cord-like structure with fimbriated end along its free edge, entered the peritoneal cavity through the ring and crossed obliquely over the bladder as a lax membrane to the opposite pelvic brim, where it was attached about the point of bifurcation of the iliac artery. it was attached about the point of bifurcation of the iliac artery. It was rather undersized, devoid of a tunica vaginalis, and on incising the tunica albuginea a normal testicular appearance was seen; the vas deferens of this testis was adherent to, and partially embedded in, a firm structure about a fingerbreadth thick, which ran upwards from the testis, through the inguinal opening, and down extraperitoneally along the left side of the bladder towards the base. In the intra-abdominal part of its course both vasa deferentia were embedded in it, the left more intimately, so that they could be dissected out only with great difficulty. When opened it was found to have a cavity admitting a fair-sized probe, the wall about a quarter of an inch thick, rugose, and apparently lined with endothelium.

The distal part was excised, about one to one and a half inches being left to avoid injury to the associated left vas deferens. The ectopic testis was removed with the accompanying fold, the cord having been freed from the above mentioned structure as far as possible, and the oblique muscles and skin sutured. wound thealed by first intention and the patient was comfortable on discharge two weeks later. examination was refused.

Serial sections of the ectopic testis, kindly made by Sir Arthur Keith, showed- only testicular tubules-no mixture of sex. "No spermatozoa are to be found and none in process of formation. The interstitial tissue is normal in amount (A. K.)."

The excessive rarity of the condition is shown by the fact that afparently only four cases of transverse ectopia are recorded.
1. Lenhossék in $\mathbf{1 8 4 5}$ first described the condition in a man of 35 with two testes in the left half of the scrotum, the right lying a little higher than the left, in front and medianward. The vasa only near the prostate.

2. Max Jordan in 1885 reported a case in a child of 8 . Ther6 were tixo testes in one patent vaginal process with one vas which divided higher up. The testes were in the left half of the scrotum and the united vasa had coalesced for the greater part of their length.

3. Berg in 1901, operating on a congenital left inguinal hernia in a boy of 13 , found two testes-one normal in a well developed scrotum, and the other, almond size, at the left ring. Both cords were intimately connected with the hernial sac; the ectopic cord had a distinct mesentery and passed up across the space of Retzius to the right inguinal region, whence it descended to the pelvis. The right ring was palpated and found closed.

4. Halstead (1907) records a case with two small testes and a large left inguinal hernia; the epididymes were fused, but the vasa deferentia were distinct up to the region of the internal ring where they were united.

\section{Note BY Sir A. Keith.}

The abnormal structure found passing upwards from the testis, and descending to the base of the bladder, can only be the result of fusion and persistence of the Müllerian ducts, forming a uterus masculinus of unusual size and persistence. If this were drawn down into the hernial sac, as is quite possible, it would bring with it the attached undescended right testis. The vasa deferentia should be embedded in the "structure," exactly as found at operation. This, I think, is the true interpretation, not a double or dichotomous condition of the left testis.

It is to be supposed, therefore, that in this patient there had been a persistence of the stage reached and usually passed through in the third month of development.

REFERENCES.

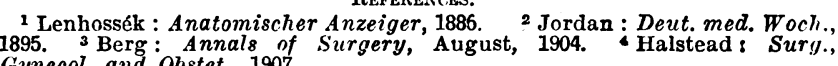
Gynecol. and Obstet., 1907.

\section{NEPHRO-URETERAL ANASTOMOSIS AFTER COM- PLETE AVULSION OF THE URE'TER.} BY

\section{R. CAMPBELL BEGG, M.C., M.D., F.R.C.S.Ed., M.Sc.N.Z.,} GENITO-URINARY SURGEON, WELLINGTON HOSPITAL, N.Z.

A man, aged 42, was referred to me on January 19th, 1926, complaining of constant pain in the right loin. It had existed with varying intensity for many years, but he had had nothing in the nature of an attack of renal colic. Investigation showed that there was a round calculus in the right renal pelvis, and that the urine was infected with $B$. coli and was delivering pus. The kidney function, however, br dye tests showed that there was only slight loss of function on the right side as compared with the left. In the latter, indigo carmine, after intravenous injection, appeared in strong concentration in five minutes, while the dye did not appear till six and a half minutes from the right side, but the concentration was good. The calculus appeared to be about $1 \mathrm{~cm}$. in diameter, so pyelo-lithotomy was advised.

\section{Operation.}

On February 12th the kidney was exposed with some difficult owing to its high position; a portion of the twelfth rib had to be resected The organ seemed healthy except for one or two points of subcapsular scarring. The pelvis was mostly intrarenal and the calculus was lying just outside the hilum, while immediately below it a ureter of normal calibre passed downwards. The calculus was removed by a vertical incision. The walls of the pelvis was removed by a thin and there was a ring of ulceration where the stone had been lying. An attempt to stitch the walls of the incision with the finest round needle and catgut resulted in ever stitch pulling through. My assistant had to exercise a certain amount of tension on the kidney to keep the field in view, and suddenly the ureter came completely away from the pelvis at the ine of the circular ulcer. The line of separation was very close to the kidney, and the little pelvic tissue available for anastomosis was so friable that an end-to-end junction was impossible; after some atlempts at this there remained no extrarenal pelvis, but merely a few bits of ragged tissue around the orifice of the renal sinus Nephrectomy was considered, but owing to the comparatively good renal function present seemed undesirable. The urete was not dilated and had good muscular walls. A No. $6 \mathrm{~F}$ catheter 